\title{
Respiratory Simulator for Robotic Respiratory Tract Treatments
}

\author{
Maria Elena Giannaccini ${ }^{1 *}$, Keren Yue ${ }^{1 *}$, James Graveston ${ }^{2}$, Martin Birchall ${ }^{3}$, Andrew Conn ${ }^{4}$ and Jonathan Rossiter ${ }^{1}$
}

\begin{abstract}
Robotic healthcare is a growing and multi-faceted field where robots help perform surgery, remotely provide care to patients, aid in supplying various physical therapies and further medical research. Robotic simulators of human physiology provide a powerful platform to advance the development of novel treatments, prostheses and therapies. This study focuses on the design, building, testing and characterisation of a novel simulator of the human respiratory system. The comparison between healthy subjects breathing and coughing physiological values and the values achieved utilising our novel bioinspired respiratory simulator shows that the latter is able to reproduce peak flow rates and volumes.
\end{abstract}

Index Terms-simulator, respiratory system, robotic healthcare, bioinspiration

\section{INTRODUCTION}

Robotic physiology simulators such as artificial hearts and prostheses provide a unique opportunity to exploit a wealth of novel technologies and pave the way for robotic advancements in healthcare. Such solutions not only help develop therapies used to better the day-to-day lives of patients by providing test-beds which reproduce the functionality of human organs but also minimise the risk to human testers.

Respiratory diseases can potentially be life-threatening, as is the case in pulmonary embolism, pneumonia, trauma, lung cancer and acute asthma. Lung cancer amounted to $13 \%$ of cancer cases in UK in 2014 [7]. Common treatments for some of these diseases include excision and tracheotomy. Hence robotic solutions are needed to provide better comfort, quality of life and outcomes.

Simulators and models of the respiratory system possess considerable utility in evaluating new robotic devices and techniques, improving our understanding of the respiratory system, guiding therapies and clinician education [9], testing/trialling new treatments or pharmaceutics. A respiratory

These authors contributed equally to this work. This research was funded by EPSRC grants EP/MO26388/1, EP/MO20460/1, EP/PO25846/1 and Elizabeth Blackwell Trust, MRC Confidence in Concepts award and Wellcome Trust Transation Award, RoboVox Soft Robotic Total Larynx Replacement.

${ }^{1}$ Department of Engineering Mathematics, University of Bristol and Bristol Robotics Laboratory, Bristol, UK. maria.elena.giannaccini, keren.yue, Jonathan.Rossiter@bristol.ac.uk@bristol.ac.uk

${ }^{2}$ Department of Mechanical Engineering, University College London, Torrington Place, London, UK j.graveston@ucl.ac.uk

${ }^{3}$ Department of OtolaryngologyHead and Neck Surgery, UCL Ear Institute, University College London, London, UK m. birchall@ucl . ac.uk

${ }^{4}$ Department of Mechanical Engineering, University of Bristol and Bristol Robotics Laboratory, Bristol, UK A. Conn@bristol . ac.uk

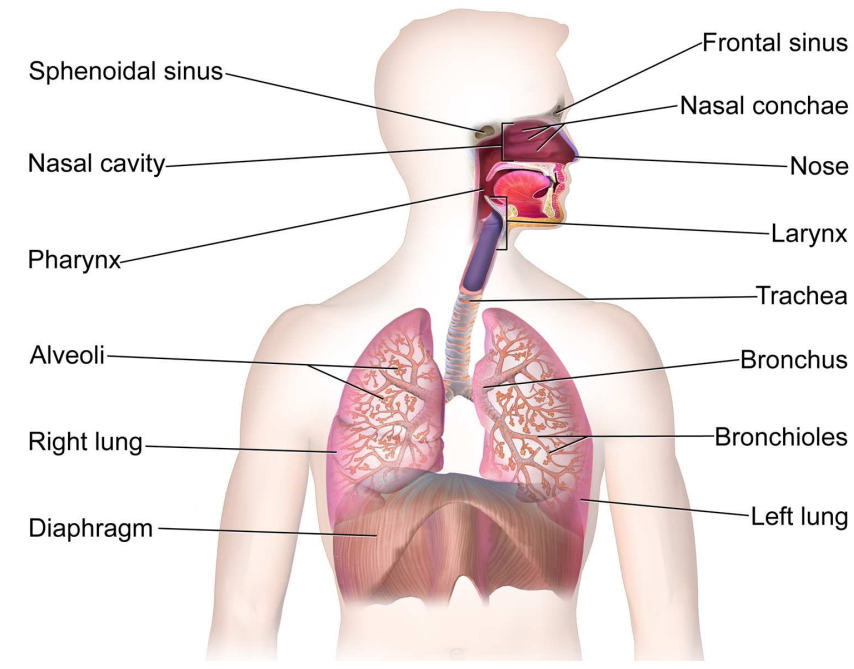

Fig. 1: Anterior view of the respiratory system [18].

simulator is either a physical or numerical platform which reproduces human respiratory patterns.

Numerical simulations of airflow for breathing have used MRI images to model the respiratory system from the nose to the upper bronchi region [20]. Other studies have used CT images and automatic meshing to compute the fluid dynamics of pulmonary gas flow [12], have concentrated on oscillatory flow in the laryngeal channel [16] or have studied power loss mechanisms in pathological tracheas [1]. On the other hand, physical apparatus that simulate the human respiratory system have been developed to characterise particle delivery within lungs [11] and to simulate specific lung pathologies [10].

None of these respiratory simulators feature anatomically correct dimensioning of respiratory system physical structures and multiple actuation systems, which are featured in our design. In this work we present a new physical simulator that aims to replicate the breathing and coughing characteristics of healthy humans, providing a first-stage evaluative tool for the development of robotic healthcare solutions for serious respiratory conditions. The accurate simulation of respiration in our benchtop platform will generate a platform for novel implantable assistive devices and advanced treatment of bronchial conditions such as asthma. 


\section{Bioinspired Robots in HealthCARE}

Bioinspiration is of extreme importance in the development of robotic platforms aimed at simulating and testing the behaviour of a biological structure. This is especially important for the respiratory system which is such a vital component of human life, and a delicate environment in which to develop robotic healthcare treatment. For this reason we designed our respiratory simulation platform so that it replicates the dimensions and shape of the anatomy of the human respiratory system as closely as possible (Fig. 1). The ability to reproduce a physiological breathing pattern is chosen as a method of testing and evaluating our simulator. First, experiments are conducted on a number of healthy subjects to establish the profile of physiological flow rate over time. Secondly, the ability of the simulator to match the breathing profiles is investigated. The experiments conducted on healthy subjects are described in Section III. A description of the simulator design and the experiments conducted on it is provided in Section IV, where a comparison of the physiological profiles with those obtained with the simulator is also provided. Conclusions and future work are detailed in Section V.

\section{Breathing And Coughing In Healthy SubJects}

\section{A. Description of Hardware}

A Sensirion SFM3000 flow meter was used to measure normal breathing and deep breathing and a BioPac Pneumotachometer (TSD137H) was utilised to gather coughing data. A disposable anti-viral and anti-bacterial filter prevented contaminants from each participant from going into the flow meter (Fig. 2). The data gathered with the Sensirion SFM3000 flow meter was collected utilising an Arduino Nano, while the Biopac system utilises its own data acquisition system and software.

\section{B. Experiments}

Breathing and coughing experiments were carried out with 8 healthy subjects within the age group of 25-40 years (ethics approval was granted from Faculty of Engineering Ethics committee, University of Bristol Ref:57763). All subjects

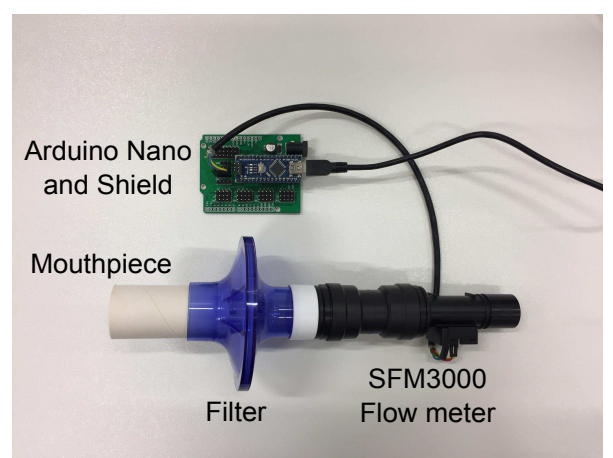

Fig. 2: Devices used for experiments with subjects. The filter is connected by an adaptor to the flow meter, which communicates with the Arduino microcontroller. followed the same experimental procedures. The subjects were asked to perform three tasks:

1) Normal Breathing (Duration: 30-60s) The subjects are asked to breath as naturally as they can.

2) Deep Breathing (Duration: 30-60s) The subjects are asked to take a series of deep breaths.

3) Coughing (Cough 3 times) The subjects are asked to take breath normally, then take a deep breath and then cough as forcefully as they can.

Before beginning each test, subjects were asked to sit down and relax for a few minutes to avoid artefacts in the measurements due to any preceding aerobic activities.

\section{Results}

The average values and relative standard deviation for peak flow rate and expiratory volume from the experiments are shown in Table I. The mean frequency is $13 \pm 3$ breaths $/ \mathrm{min}$ for normal breathing and $7 \pm 2$ breaths/min for deep breathing.

\begin{tabular}{lll}
\hline & Peak Flow Rate $(\mathrm{L} / \mathrm{min})$ & Expiratory Volume $(\mathrm{L})$ \\
\hline Breathing & $15.12 \pm 3.18$ & $0.46 \pm 0.15$ \\
Deep Breathing & $59.32 \pm 22.69$ & $1.40 \pm 0.34$ \\
Coughing & $538.42 \pm 109.70$ & $1.24 \pm 0.18$ \\
\hline
\end{tabular}

TABLE I: Peak Flow Rate and Expiratory Volume obtained from experiments

The flow rate values for breathing and for coughing found in these experiments match the values reported in the literature [15], [2]. In order to test the ability of our simulator in reproducing human physiological breathing and coughing patterns, an example of both from the set of data acquired was chosen. The representative example chosen for the normal breathing experiment with human subjects is shown in Fig. 5 and the representative example chosen for the deep breathing experiment with human subjects is shown in Fig. 7. The simulator was then programmed to reproduce each of these patterns.

As can be observed from Table I, Fig. 5 and Fig. 7, the flow rate magnitude $(60 \mathrm{~L} / \mathrm{min})$ for deep breathing is approximately four times that of normal breathing $(15 \mathrm{~L} / \mathrm{min})$. The coughing profile can be seen in Fig. 11.

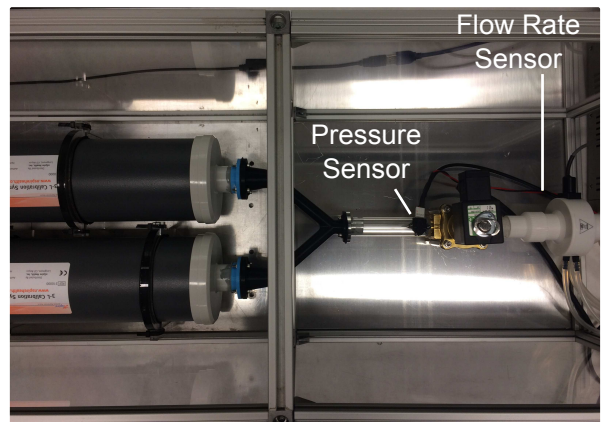

Fig. 3: Top view of the respiratory simulator system. 


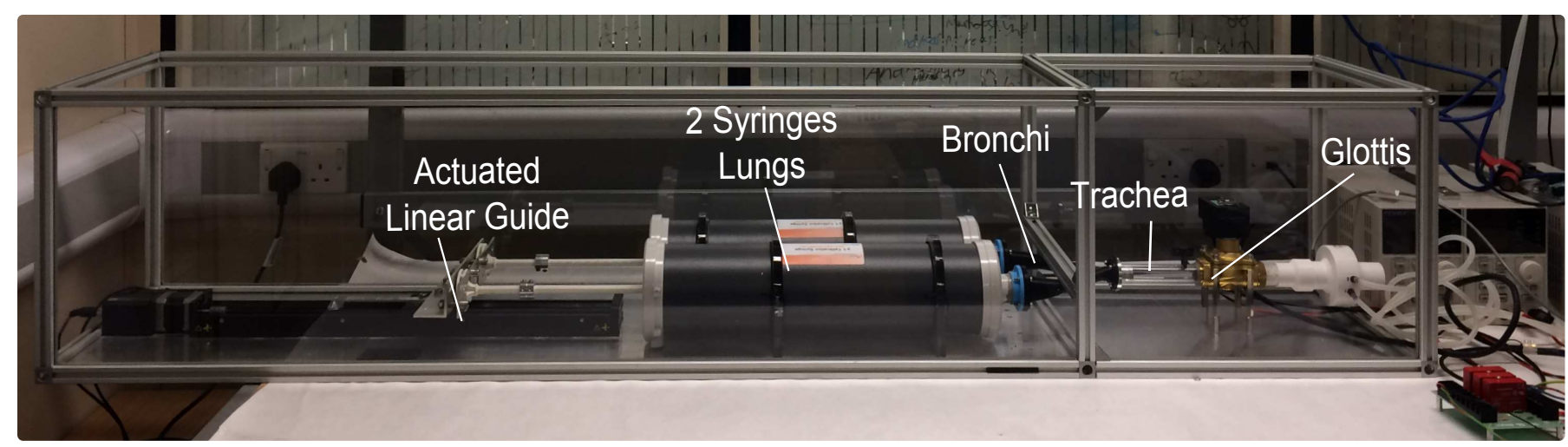

Fig. 4: Lateral view of the respiratory simulator system.

\section{RESPIRATORY SimUlator}

The respiratory simulator is designed by replicating as closely as possible the dimensions, shape and function of the human respiratory system in the hardware design. Each human lung holds approximately three liters of air and this is replicated in our system by two 3 liter syringes (see Fig. 3). Anatomy literature is utilised to identify the average anatomically-correct value for the angle of tracheal bifurcation (approximately 70 degrees) [19], [14], [6], length (the average length of the left bronchus is $47.8 \mathrm{~mm}$ for men and $43.5 \mathrm{~mm}$ for women and the average length of the right bronchus is $15.3 \mathrm{~mm}$ for men and $13.2 \mathrm{~mm}$ for women) and diameter (the average diameter of bronchi in men is $12.9 \mathrm{~mm}$ in the left bronchus and $15.2 \mathrm{~mm}$ in the right bronchus, the average diameter of bronchi in women is $10.1 \mathrm{~mm}$ in the left bronchus and $12.5 \mathrm{~mm}$ in the right bronchus) [13] of the simil-bronchi component of our simulator. Similarly, the dimensions of the trachea component of the simulator are matched to the values (average diameter $17.07 \mathrm{~mm}$ and average length $107.9 \mathrm{~mm}$ ) found in anatomy literature [3], [5], [17] and [8]. Hence the simulator system is designed to approximate a 1:1 replica of the human respiratory system. Naturally, approximations are made to simplify the physical design of the structure. One of the main approximations made is that all components of the simulator are rigid, contrary to the nature of the human respiratory system, which is prevalently composed of soft and compliant structures.

\section{A. Description of Hardware}

The simulator comprises five components: actuation system, lungs, bronchi, trachea and glottis (Fig. 4). The actuation system is an actuated linear guide (Zaber X-LSQ450B-E01) fixed to the plungers of two $3 \mathrm{~L}$ calibration syringes (KoKo KRS007) which imitate the function of lungs. Two flange connectors secure the right hand side of the syringes to the PLA 3D printed channels that imitate the bronchi. The channels gradually reduce their diameter to the physiological dimension of $12.7 \mathrm{~mm}$ and converge into one channel at a 70 degrees angle between the two channels (which mirrors the tracheal bifurcation, its physiological counterpart). This simil-bronchi component is $29 \mathrm{~mm}$ long. A further flange connector connects the simil-bronchi component to a $19 \mathrm{~mm}$ internal diameter, $108 \mathrm{~mm}$ long acrylic tube which imitates the function of the trachea. A differential pressure sensor (SIP, NB 015PD TruStability, Honeywell) is inserted into a hole in the simil-trachea. A normally open, two port, solenoid valve (SCE210C35-24VCC, Asco) imitates the opening and closing function of the glottis. The TSD13717 BioPac flow meter is fixed to the exhaust side of the solenoid valve. The valve is powered through a relay which is controlled using a DAQ (USB-6211, National Instruments), which also collects the measurements from the pressure sensor. The system is controlled via MATLAB2017®, which is also used for the data post-processing.

The working principle of the simulator is repeatable and robust: the computer-controlled actuated linear guide is used to replicate the movement of the human diaphragm and generates the force needed to drive the syringes. The air is then pushed through the simil-bronchi and simil-trachea components. In the case of breathing, the solenoid valve is kept open and the breathing flow rate is measured with the BioPac flow meter attached to the exhaust side of the valve. The simulation of a cough comprises four stages: 1) the solenoid valve is closed, 2) the actuated linear guide compresses air in the system until 3) the pressure sensor registers a pressure above a fixed threshold; this triggers 4) the opening of the valve which causes the exhalation of the compressed air. The peak flow rate is measured by the BioPac flow meter.

\section{B. Breathing Experiments}

The tidal volume (TV, volume of air displaced between inhalation and exhalation in normal breathing) is utilised as a starting point in simulating the human breathing pattern. TV is calculated by identifying the flow rate data obtained in the experiments described in Section III. Since the volume of the syringes is known, a simple proportion yields the syringe plunger travel needed to obtain the same volumetric flow as in the physiological experiment. Flow rates and acceleration are identified utilising an iterative process. 


\section{Results}

Fig. 6 shows simulated flow profiles replicating the normal breathing in Fig. 5. Fig. 8 shows simulated flow profiles replicating deep breathing in Fig. 7. The simulator is able to reproduce both the breathing TV of $0.35 \mathrm{~L}$ and deep breathing TV of $1.5 \mathrm{~L}$. The complexity of the physiological profile is not fully reproduced by the simulator.

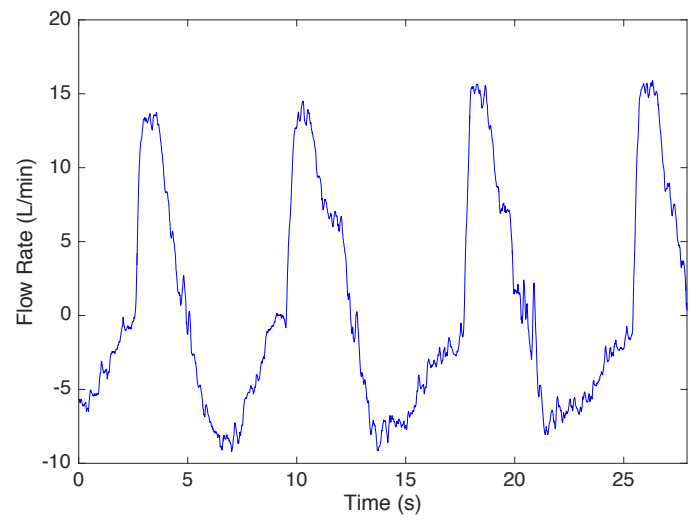

Fig. 5: Normal human breathing.

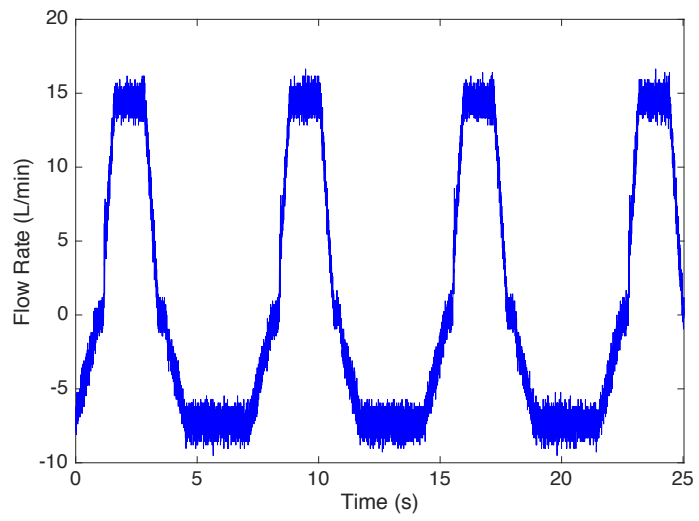

Fig. 6: Simulator breathing.

However, the maximum flow rate is replicated in both experiments, together with the inspiration and expiration duration. The overall flow profile provides the important prototypical and highly repeatable respiratory function needed for robotic device development and testing. The simulator successfully attains the reproduction of a simplified breathing and deep breathing pattern.

\section{Coughing Experiments}

Coughing is a defence mechanism comprising three phases: (i) an inspiratory phase, (ii) a forced expiration against a closed glottis, (iii) opening of the glottis followed by fast exhalation, which results in a cough sound [4]. Experiments aimed at reproducing coughing are conducted by specifying the travel distance, velocity and acceleration for the linear guide, which

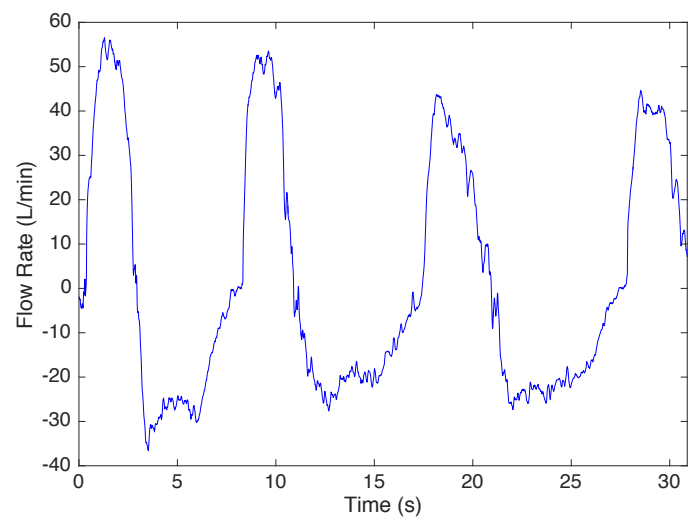

Fig. 7: Human deep breathing.

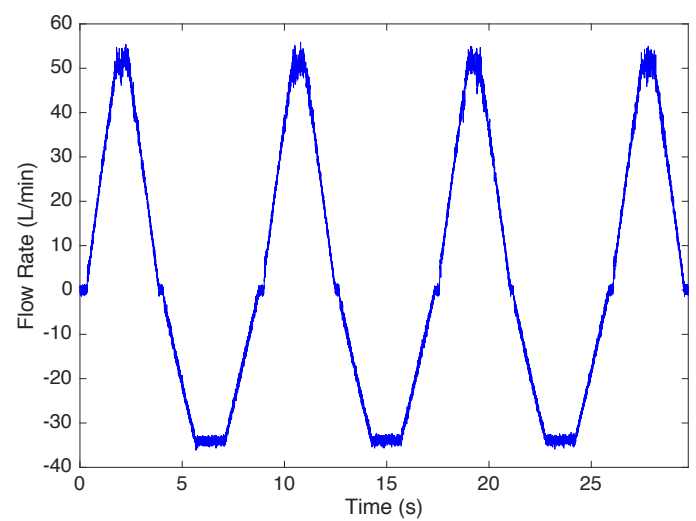

Fig. 8: Simulator deep breathing.

pushes the syringes forward and increases the internal system pressure. Once a certain pressure threshold is reached, the solenoid valve is opened, thus releasing the pressurised air and generating an artificial cough.

In order to reproduce a coughing event, the three most important variables are: volume, flow rate magnitude and cough duration. The coughing experiments consist of three stages:

1) In the first stage, a series of threshold pressures is tested to characterise the relationship between flow rate and threshold pressure. The acquired characterisation is used to inform the following experiment, where the desired flow rate magnitude is attained by setting the relative pressure threshold.

2) In the second stage, the volume of the typical human cough acquired in Section III is determined and a simulation of a cough of similar air volume is performed, hence volume is kept constant. The flow rate is also kept constant.

3) In the third stage, the cough duration and maximum flow rate (but not the total volume) are kept constant between the physiological cough and the simulated one. 


\section{E. Results}

Fig. 9 shows a monotonic relationship between pressure threshold and flow rate magnitude. Fig. 10 shows internal system pressure profile and flow rate for a typical simulated cough. As soon as the threshold pressure $\left(P_{T}\right)$ of $93.08 \mathrm{mbar}$ is reached, the solenoid valve is opened and this generates a flow impulse of approximately $100 \mathrm{~ms}$ followed by a tail of lower flow for approximately $200 \mathrm{~ms}$.

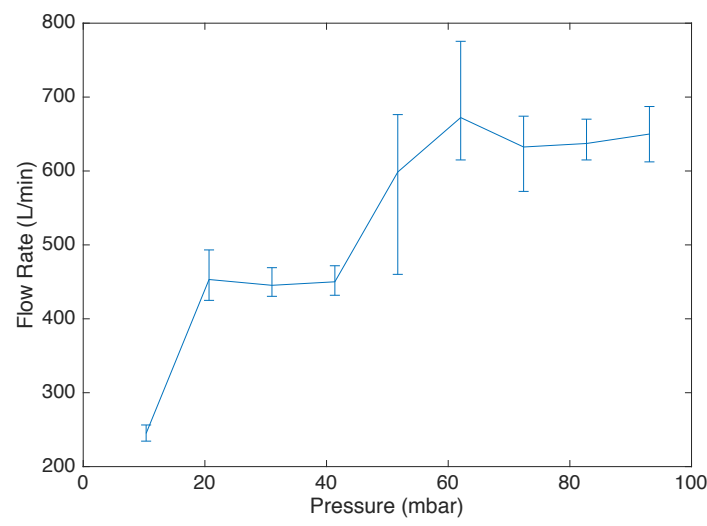

Fig. 9: Relationship between pressure threshold and flow rate magnitude.

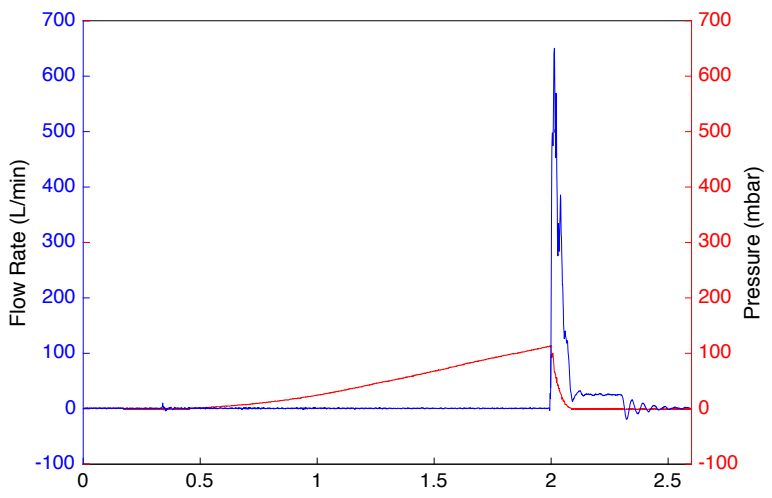

Fig. 10: Correlation between internal system pressure profile and flow rate.

In the constant volume tests (second stage, Fig. 12) the simulator is able to replicate a cough event of comparable volume to the physiological cough (Fig. 11). Although the simulator cough profile is over a much longer duration, the maximum flow rate of the two coughs is at $650 \mathrm{~L} / \mathrm{min}$.

The cough flow profile (Fig. 13) is most successfully reproduced in the results of the third stage experiment, shown in Fig. 14. In this case the total simulated cough event duration $(0.4 \mathrm{~s})$ is similar to the human subject one, but less highmagnitude flow $(0.1 \mathrm{~s}-0.35 \mathrm{~s})$ is shown.
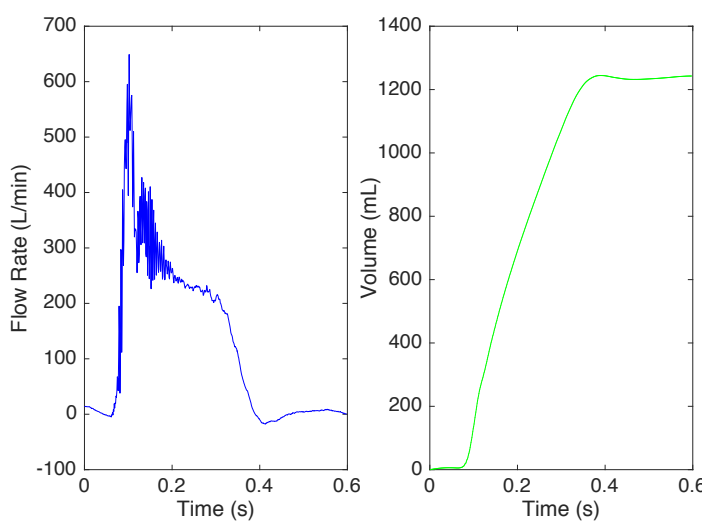

Fig. 11: Human cough constant volume.
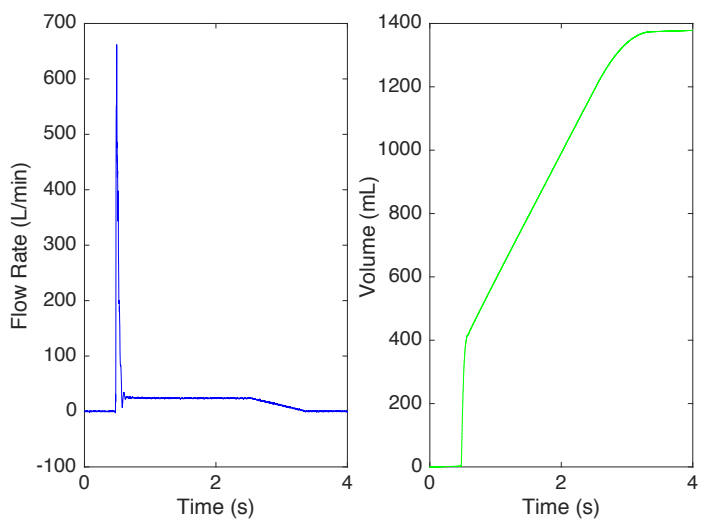

Fig. 12: Simulator cough constant volume.

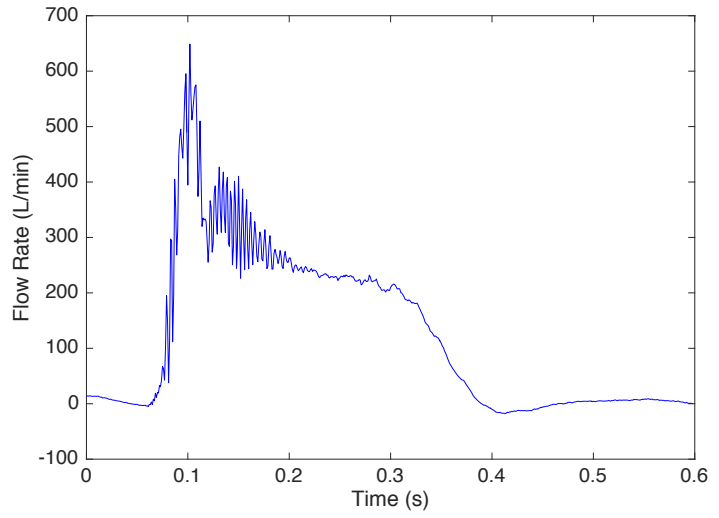

Fig. 13: Human cough constant time. 


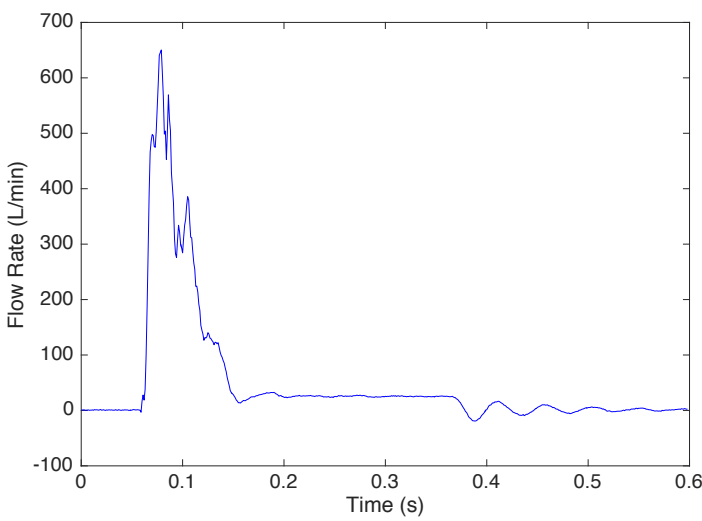

Fig. 14: Simulator cough constant time.

\section{CONClusion}

The robotic simulator presented in this work is able to reproduce the frequency, tidal volume and amplitude of the physiological flow rate profiles of breathing and deep breathing. However, the novel simulator is not able to reproduce the higher frequency flow artifacts present in the breathing profiles observed. It cannot be excluded that some of the artifacts observed in the physiological breathing profiles are due to the compliant nature of the human respiratory system and the characteristics and orientation of the muscles driving the human diaphragm and chest. Regarding the coughing simulation, the system described in this paper is able to reproduce the volume, flow rate magnitude and duration of a human cough. Also in this case, human cough profiles are not exactly reproduced. A more advanced control system is being prepared to tackle the issue. Also, it is possible that some of those non-linearities are the result of the role of the larynx, glottis and upper airway on coughing. Hence designing and building soft robotic devices to mimic the naturally compliant respiratory anatomy is one of our future aims to make our platform even more suitable to testing and investigating the human airway system.

\section{ACKNOWLEDGMENT}

The authors would like to acknowledge the help of the subjects who took part in the experiments and the Faculty of Engineering Ethics committee.

\section{REFERENCES}

[1] A. J. Bates, A. Comerford, R. Cetto, R. C. Schroter, N. S. Tolley, and D. J. Doorly. Power loss mechanisms in pathological tracheas. Journal of Biomechanics, 49(11):2187-2192, 2016.

[2] A Beardsell, S Bell, S Robinson, and H Rumbold. Mcem part a: Mcqs. Royal Society of Medicine Press, page 2009, 2009.

[3] E. Breatnach, G. C. Abbott, and R. G. Fraser. Dimensions of the normal human trachea. American Journal of Roentgenology, 142(5):903-906, 1984.

[4] Kian Fan Chung and Ian D Pavord. Prevalence, pathogenesis, and causes of chronic cough. The Lancet, 371(9621):1364-1374, 2008.

[5] Ugur Cinar, Smeih Halezeroglu, Erdal Okur, Mehmet Akif Inanici, and Semra Kayaoglu. Tracheal Length in Adult Human : The Results of 100 Autopsies. International Journal of Morphology, 34(1):232-236, 2016.

[6] V Coppola, G Vallone, E Coscioni, M Coppola, G Maraziti, M Alfinito, and G Di Benedetto. [Normal value of the tracheal bifurcation angle and correlation with left atrial volume]. La Radiologia medica, 95(5):461-5, may 1998.

[7] http://www.cancerresearchuk.org. Lung cancer incidence statistics, $\mathrm{Au}-$ gust 282014.

[8] Kirollos Salah Kamel, Gabriel Lau, and Mark D Stringer. In vivo and in vitro morphometry of the human trachea. Clinical Anatomy, 22(5):571579, 2009.

[9] Neil R Macintyre and Md Faarc. Respiratory System Simulations and Modeling Introduction Computerized Simulation of Patient Signs and Symptoms Computerized Anatomic Simulation and Modeling of the Respiratory System Simulating the Airways Parenchymal and Mediastinal Modeling and Simulati. Respir Care, 49(4):401-408, 2004.

[10] S. Mesic, R. Babuska, H.C. Hoogsteden, and A.F.M. Verbraak. Computer-controlled mechanical simulation of the artificially ventilated human respiratory system. IEEE Transactions on Biomedical Engineering, 50(6):731-743, jun 2003.

[11] N.M. Minskoff. Respiratory system simulator, July 28 2016. US Patent App. 15/004,865.

[12] Shinjiro Miyawaki, Eric A. Hoffman, and Ching Long Lin. Numerical simulations of aerosol delivery to the human lung with an idealized laryngeal model, image-based airway model, and automatic meshing algorithm. Computers and Fluids, 148:1-9, 2017.

[13] C Mrudula and M Krishnaiah. The study of bronchial tree. International journal of pharma and bio sciences, 2(1):166-172, 2011.

[14] By S Nianuchehr Alavi, Theodore E Keats, Williani NI O, and NIl CHARLOTTESVIIIE. THE ANGLE OF TRACHEAL BIFURCATION: ITS NORMAL MENSURATION*. 1970.

[15] AJ Nunn and I Gregg. New regression equations for predicting peak expiratory flow in adults. $B M j, 298(6680): 1068-1070,1989$.

[16] Christine Renotte, Vincent Bouffioux, and Frédéric Wilquem. Numerical 3D analysis of oscillatory flow in the time-varying laryngeal channel. Journal of Biomechanics, 33(12):1637-1644, 2000.

[17] Shigeki Sakuraba, Ryohei Serita, Junya Kuribayashi, Shizuko Kosugi, Hirofumi Arisaka, Kazuichi Yoshida, and Junzo Takeda. Comparison of tracheal diameter measured by chest $\mathrm{X}$-ray and by computed tomography. Anesthesiology Research and Practice, 2010:3-6, 2010.

[18] Blausen.com Staff and Blausen.com Staff. Medical gallery of Blausen Medical 2014, 2014.

[19] Paul Stark. Radiology of the trachea. Thieme Medical Publishers, 1991.

[20] X. Y. Xu, S. J. Ni, M. Fu, X. Zheng, N. Luo, and W. G. Weng. Numerical investigation of airflow, heat transfer and particle deposition for oral breathing in a realistic human upper airway model. Journal of Thermal Biology, (May), 2016. 